\title{
ECOC Random Fields for Lumen Segmentation in Radial Artery IVUS Sequences
}

\author{
Francesco Ciompi ${ }^{1,2}$, Oriol Pujol ${ }^{1,2}$, Eduard Fernández-Nofrerías ${ }^{3}$, \\ Josepa Mauri ${ }^{3}$, and Petia Radeva ${ }^{1,2}$ \\ 1 Dep. of Applied Mathematics and Analysis, University of Barcelona, Spain \\ 2 Computer Vision Center, Campus UAB, Bellaterra, Barcelona, Spain \\ 3 University Hospital "Germans Trias i Pujol", Badalona, Spain \\ f.ciompi@cvc.uab.es
}

\begin{abstract}
The measure of lumen volume on radial arteries can be used to evaluate the vessel response to different vasodilators. In this paper, we present a framework for automatic lumen segmentation in longitudinal cut images of radial artery from Intravascular ultrasound sequences. The segmentation is tackled as a classification problem where the contextual information is exploited by means of Conditional Random Fields (CRFs). A multi-class classification framework is proposed, and inference is achieved by combining binary CRFs according to the Error-CorrectingOutput-Code technique. The results are validated against manually segmented sequences. Finally, the method is compared with other state-ofthe-art classifiers.
\end{abstract}

\section{Introduction}

In order to evaluate the effects of drugs and vasodilators administration, the morphological alteration of the artery must be analyzed after the drug treatment. The most indicative parameter that can be used to evaluate the drug effectiveness is the change of lumen volume. Intravascular Ultrasound (IVUS) is an imaging technique that allows to explore both arterial vessel morphology and composition; as such, it is suitable to perform the required measurement.

Most of the proposed approaches for automatic lumen segmentation devolve upon active contour models the organization and interpolation of image features belonging to the vessel border. Ad-hoc solutions, statistical and probabilistic models including a-priori knowledge of the vessel geometry are also considered. In [12] the gray level probability density function of the vessel structures, following Rayleigh distribution, is used. In [3] a cost function over the edge image of the vessel is defined, to perform a graph search based approach in which each pixel is a node, determining the border as the minimum cost path. In 4 a shape space is constructed from statistical analysis of morphology on training data and borders are constrained to a smooth closed geometry. In [5] the lumen detection is achieved by classifying blood areas using Adaboost.

The appearance of the lumen morphology in IVUS images is often corrupted, mainly due to the effect of the guide of the catheter and calcifications in the 


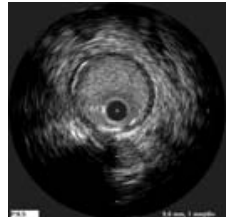

(a)

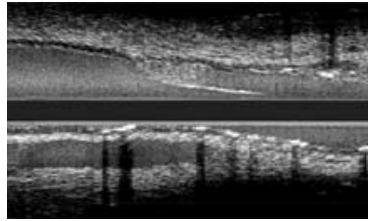

(b)

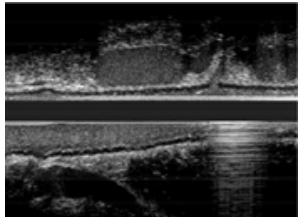

$(c)$

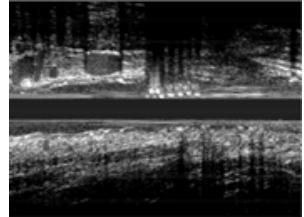

$(d)$

Fig. 1. Short-axis IVUS image (a). Examples of corrupted areas in IVUS longitudinal cuts: presence of outer arteries or veins (b), bifurcations and texture distortions (c), calcifications and lack of continuity in vessel structure $(\mathrm{d})$.

vessel wall. Furthermore, the presence of bifurcations or outer vessels, apparently identical to the lumen area, can lead to an erroneous segmentation, especially when the border appearance is subtle (see Fig. 11). Some of the existing automatic lumen detectors cope with these problems by including a-priori knowledge or spatial constrains related to the geometrical morphology of the vessel; other methods perform a pre-emptive detection of calcifications, bifurcations or guide effects, in order to predict where a correction on vessel geometry has to be applied.

The method proposed in this paper is intended to overcome the need of ad-hoc corrections or case-specific knowledge by formulating the segmentation process as a classification problem, exploiting the contextual information over the vessel geometry. The contextual information helps the inference in ambiguous cases by exploiting the relationships among connected image points. To exploit this, we propose to apply Conditional Random Fields (CRFs) [6], a discriminative graphical model that estimates an a-posteriori probability function by considering the observation of each node in the graph and both predictions and observations of nodes in its neighborhood. The use of a CRF model as basic classifier allows to design a framework in which different classes, together with their spatial relationship, can be learnt from a set of labeled examples. In order to differentiate the lumen area from the rest of the vessel structure, usually corrupted by the phenomena discussed above, we design a multi-class model where lumen, vessel interfaces and outer tissue are represented as separate classes in a supervised learning framework. The multi-class definition of the problem is here solved by means of the Error-Correcting-Output-Code (ECOC) [7] technique. Finally, the whole lumen structure detected by the ECOC Random Field (ECOC-RF) is delineated by an active contour model.

Summarizing, the novelty of the proposed methodology consists in defining the lumen detection and segmentation as a multi-class classification problem using an ECOC-based supervised framework. Moreover, in order to cope with the ambiguity of local image configuration, we take profit of the image context by using $\mathrm{CRF}$ as a classifier able to make inference basing on local image features and neighborhood relationships. Finally, the vessel region is regularized by a deformable model. 


\section{Methodology}

\subsection{Classes Definition}

Looking at the longitudinal cut, different structures can be observed: blood region, interfaces and the outer part of the vessel. The class defined as interfaces actually consists in the intima, typically identified by a transition from the darker blood texture to a brighter area, and media-adventitia interfaces, characterized by a dark band separating the internal vessel membrane from the outer tissue. Given the different appearance of these interfaces as well as the fact that they can be visible only in some regions of the image, we split the interface class into two sub-classes. Since even the manual segmentation of interfaces is difficult, the sub-classes definition (border 1 and border $_{2}$ ) is based on a $k$-means unsupervised classifier [8]. The unsupervised clustering is solely used to define the two subclasses before the training process. Therefore, a 4 classes classification problem is defined.

\subsection{Features Extraction for Conditional Random Fields}

$\mathrm{CRFs}$ as binary classifier requires a graphical representation $\mathcal{G}=(\mathcal{S}, \mathcal{E})$ (lattice) of the input data, where $\mathcal{S}$ is the set of nodes, corresponding to each block of the lattice and $\mathcal{E}$ indicates edges, corresponding to the interconnections among nodes. In this case, the lattice is constructed by dividing the longitudinal cut into squared blocks of fixed size $W$. Features are first extracted from the whole image, then a feature vector $\mathbf{x}_{i}$ is assigned to each node $\mathcal{S}_{i} \subset \mathcal{S}$ by considering the median value of each feature in the block. The feature selection process proposed in [5] has been used to select, among a wide set of texture descriptors, the most discriminant features for the proposed problem, resulting in Gabor filters 9], Local Binary Patterns 10, Sobel filter in the $\hat{x}$ direction, mean value, standard deviation and the ratio among these two values on the grey-levels of the image, computed by a sliding windows of size $H$. Finally, the First Order Absolute Moment (FOAM) of the grey levels [11] is also used. FOAM is an operator that computes a vector always pointing towards the strongest gray-level discontinuity and assuming magnitude close to zero when applied to the discontinuity itself. Features extraction process results in a 10-dimensional observation vector.

\subsection{Conditional Random Fields}

Once the graph has been constructed, the observation vector $\mathbf{x}_{i} \in X$ and the label $y_{i} \in Y=\{-1,+1\}$ can be assigned to each node $\mathcal{S}_{i}$. The probability function modeled by CRFs for a given binary classification problem can be estimated as:

$$
P(y \mid \mathbf{x}, \theta)=\frac{1}{Z(\mathbf{x})} \exp \left(\sum_{i \in \mathcal{S}} A\left(y_{i}, \mathbf{x}, \theta_{S}\right)+\sum_{i \in \mathcal{S}} \sum_{j \in \mathcal{N}_{i}} I\left(y_{i}, y_{j}, \mathbf{x}, \theta_{E}\right)\right),
$$

where $A$ is the association potential, modeling the relationship among the observation $\mathbf{x}$ from an image region (node) with the label $y$ for the region, while $I$ is 
the interaction potential, modeling the relationship among different nodes. $\mathcal{N}_{i}$ is the neighborhood of the node $\mathcal{S}_{i}$ and $\theta=\left\{\theta_{S} \cup \theta_{E}\right\}$ is a parameters vector that has to be estimated; $Z(\mathbf{x})$ is an observation-dependent normalization function. A typical estimation of the parameter vector is given by $\theta^{*}=\operatorname{argmax}_{\theta} P(y \mid \mathbf{x}, \theta)$.

\subsection{Error Correcting Output Code}

The presented CRF is able to model a conditional probability for a binary classification problem, while 4 classes are foreseen in our approach. Error Correcting Output Code [7] is a technique that combines $N$ binary classifiers to solve a $K$-classes classification problem. For each class a particular codeword $c_{k}=\{1,0,-1\}^{1 \times N}$ is obtained $(k=1, \ldots, K)$. Based on a chosen coding strategy, a matrix $\mathbf{M}$ is designed, in which each column represents a binary classifier (dichotomy) and each row represents a class. A value 1 in position $\mathbf{M}(k, j)$ means that the $j^{\text {th }}$ dichotomy classifies an unknown example as belonging to the class $k$, a value -1 means that the it belongs to the class $q \neq k$ and a 0 value means that we do not care about classification result, regarding class $k$. Therefore, to classify an unknown example, the distance between the obtained codeword and each row $m_{k}$ of the matrix $\mathbf{M}$ is computed: the inferred class will be the value $k$ reporting the minimum distance. The number of classifier, using the one-versusone coding technique is $K(K-1) / 2$. In a 4 classes problem, 6 binary classifiers must be trained (see Fig. 2).

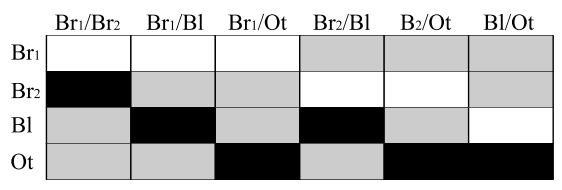

Fig. 2. Error-Correcting-Output-Codes matrix used in a 4 classes (rows) classification problem using 6 binary classifiers (columns) with one-vs-one coding technique. A white box represents a 1 value, a grey box represents a 0 value and a black box a -1 value. $B r_{1,2}=$ border $_{1,2}, \mathrm{Bl}=$ blood, Ot $=$ outer tissue.

\subsection{ECOC Random Fields for Automatic Lumen Detection}

Given the graphical representation of the input images, following [12] we define the node features vector $\mathbf{h}_{i}=\left[1, \mathbf{x}_{i}\right]$ and the edge feature vector $\mathbf{h}_{i j}=$ $\left[1,\left|\mathbf{x}_{i}-\mathbf{x}_{j}\right|\right]$, where $\mathbf{x}_{i}$ and $\mathbf{x}_{j}$ are the observations for the nodes $\mathcal{S}_{i}$ and $\mathcal{S}_{j}$, respectively. Since the classes border 1 and border 2 result from an unsupervised clustering process, no spatial relationships is expected. For this reason, the discrimination among these two classes is devolved upon an AdaBoost classifier 13 trained with examples defined by the $k$-means unsupervised classifier. Conditional Random Fields are instead trained to learn spatial dependencies on the other binary problems. Following [1214, potential functions are defined as $A\left(y_{i}, \mathbf{x}\right)=\exp \left(y_{i} \theta_{N}^{T} \mathbf{h}_{i}\right)$ and $I\left(y_{i}, y_{j}, \mathbf{x}\right)=\exp \left(y_{i} y_{j} \theta_{E}^{T} \mathbf{h}_{i j}\right)$. 
The training process basically consists in estimating $\theta^{*}=\operatorname{argmax}_{\theta} P(y \mid \mathbf{x}, \theta)$ or, equivalently $\theta^{*}=\operatorname{argmin}_{\theta} \mathcal{L}(\theta)$, where $\mathcal{L}(\theta)$ is the negative Log-posterior of the parameters given the data and the labels (see [12] for details); $\theta_{N}$ is initialized by logistic regression, while $\theta_{E}$ is empirically initialized to a constant value 0.1 .

The inference on the whole graph is performed by Loopy Belief Propagation 15. for each binary CRF model, thus generating, together with the result provided by AdaBoost, a 6 elements codeword. The definition of different areas of the image is achieved by decoding the codeword using Attenuated Euclidean Distance [16]. Lumen regions are thus defined and inference for each node is implicitly related to the contextual information of the neighborhood.

In order to achieve the final lumen segmentation, an active contour model is applied on the vessel structure provided by the ECOC-RF classification, using a gravity map as external energy function [17. This step is necessary in order to regularize the prediction by filtering some spurious points and, most importantly, by including long term interactions among classified regions. In this way, the local estimation, assumed to be robust due to the exploited contextual information, is constrained by nodes in the neighborhood and by farther nodes as well. It is worth to note that, differently from classical proposed approach, the active contour model is here applied on a higher information level, i.e. the segmentation obtained by the classification rather than the grey level information provided by the image. In our case the role of the active model consists in interpreting the results proposed by the multi-class classification rather than to infer a solution.

\section{$3 \quad$ Validation and Results}

A wide set of IVUS images sequences from radial arteries have been acquired at the University Hospital "German Trias i Pujol" (Badalona, Spain); 5 study cases, presenting the more challenging segmentation have been selected, consisting in 10 sequences. Since the amount of data and the vessel structures variety in a single sequence is large, the used data set is highly representative. Longitudinal cuts have been extracted from each sequence and lumen area has been segmented by two experts; areas in which both segmentations agreed have been considered as ground truth. Textural features described in section 2.2 are extracted using the following parameters: $(W, H)=(5,100) \mathrm{px},\left(\sigma_{\text {gabor }}, \phi_{\text {gabor }}, F_{\text {gabor }}\right)=$ $\{(12.7205,0.0442,0) ;(6.3602,0.0442,0) ;(3.1801,0.3536,3 \pi / 4) ;(1.5901,0.3536,3 \pi / 4)\}$, $\left(R_{L B P}, P_{L B P}\right)=(4,32)$ and $\left(\sigma_{1_{F O A M}}=\sigma_{3_{F O A M}}\right)=15$ px. We follow the LeaveOne-Patient-Out cross-validation technique. At each fold, manual and automatic segmentation are compared and the error in lumen area detection is computed. For each point $x$ of the border delimiting the lumen area, the error $\Delta_{B}(x)=m_{B}(x)-a_{B}(x)$ is computed; $m_{B}(x)$ and $a_{B}(x)$ are the manual and automatic border detection, respectively. Evaluated parameters are: $\max \left|\Delta_{B}(x)\right|$, mean value and the standard deviation of $\Delta_{B}(x)$. Furthermore, contiguous patches corresponding to $1.5 \mathrm{sec}$ of observations $(\simeq 0.8 \mathrm{~mm})$ have been considered in each sequence and lumen area in both manual and automatic 

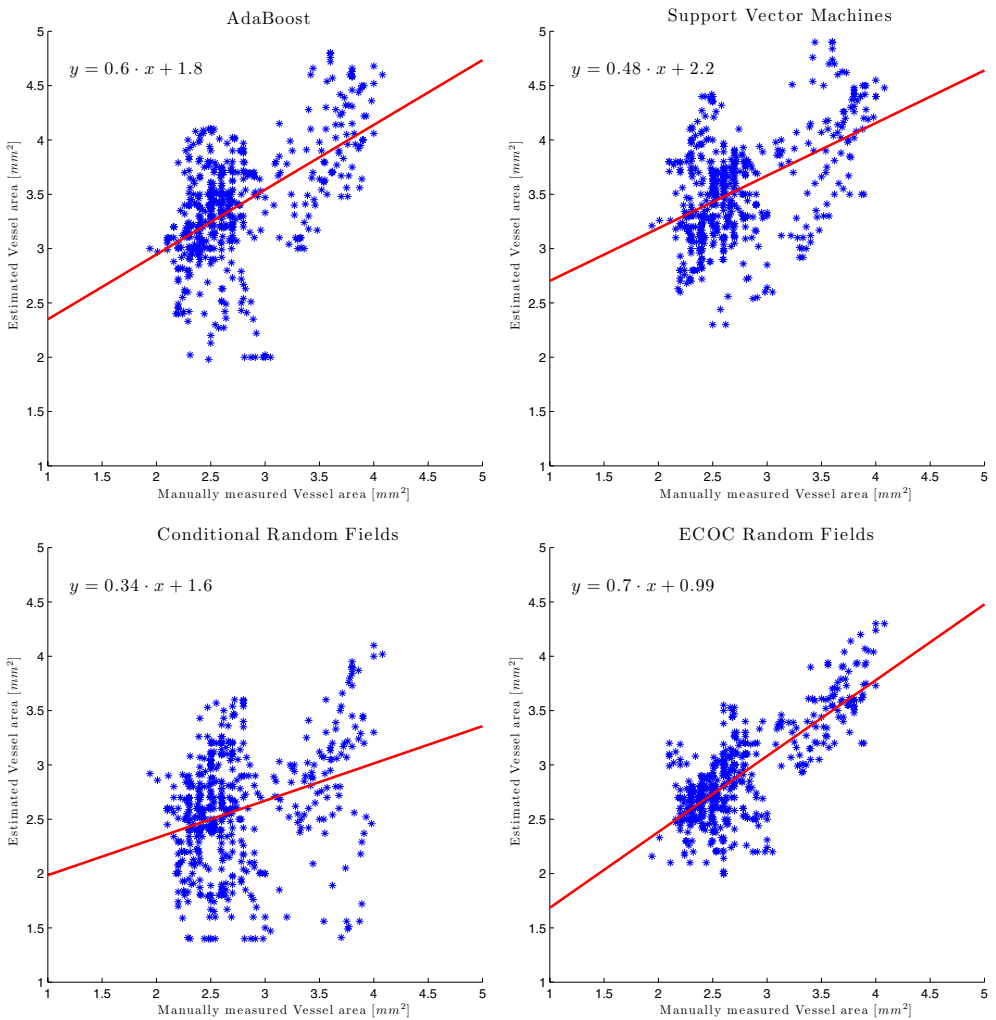

Fig. 3. Manual vs Automatic lumen area measurement, in $\mathrm{mm}^{2}$, for the classifiers considered in the comparison. The straight line fitting points is also indicated.

segmentation have been computed (Fig. 3). The correlation coefficient $\rho$ between the automatically predicted area and the ground truth area is also computed (see Table 11).

Given the classification-based nature of the proposed method, a comparison with other state-of-the-art classifiers is straightforward. For this reason, we also classified the lumen region by using AdaBoost [13] and Support Vector Machine (SVM) 18 binary classifiers in the blood-vs-outer tissue problem. Furthermore, a single binary CRF model for the same binary problem has been considered in the comparison. AdaBoost classifier has been trained with up to 50 Decision Stumps while the SVMlight 1 implementation with RBF kernel has been used for SVM $(\gamma$ and $C$ have been tuned according to [19]). CRF has been trained by Stochastic Gradient Descent [12] (batch size $=6, \eta=10^{-6}$ ). Table11reports the performance parameters for the considered methods. Figure 4 shows some examples of lumen detection in the critical cases achieved by the ECOC-RF proposed method.

\footnotetext{
${ }^{1}$ http://svmlight.joachims.org/
} 
Table 1. Results of compared methods

\begin{tabular}{|r|c|c|c|}
\hline & $\Delta_{B}(\mid$ mean $\mid \pm$ std $)$ & $\max \left(\left|\Delta_{B}\right|\right)$ & $\rho$ \\
\hline \hline AdaBoost & $0.32 \pm 0.32 \mathrm{~mm}$ & $1.3 \mathrm{~mm}$ & 0.54 \\
\hline SVM & $0.40 \pm 0.33 \mathrm{~mm}$ & $1.3 \mathrm{~mm}$ & 0.49 \\
\hline CRF & $0.08 \pm 0.41 \mathrm{~mm}$ & $0.8 \mathrm{~mm}$ & 0.31 \\
\hline ECOC-RF & $0.08 \pm 0.24 \mathrm{~mm}$ & $1.0 \mathrm{~mm}$ & 0.77 \\
\hline
\end{tabular}
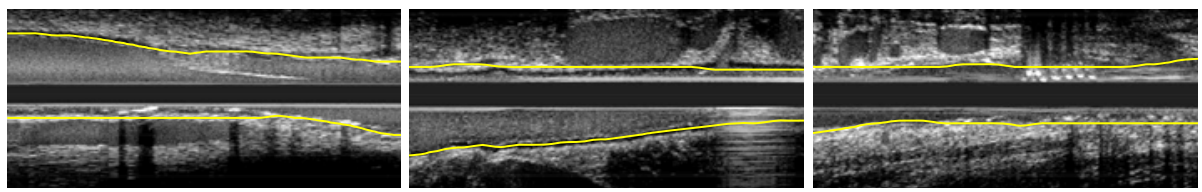

Fig. 4. ECOC-RF lumen segmentation results on the challenging examples of Fig. 1

\section{Discussions and Conclusions}

The proposed ECOC-RF method outperforms the other considered approaches (see Table 1). We can observe that a single CRF achieves a mean segmentation error lower than AdaBoost and SVM, although attaining a higher standard deviation. ECOC-RF solves this problem, thanks to the strength given by the multi-class definition. Moreover, a high correlation among computed lumen areas and ground truth is exhibited. It is worth to note that the clouds of point in the ECOC-RF case (see Fig. 3) is more compact and close to fitting straight line, thus showing much higher correlation between manual and automatic measurements in the proposed method respect to other strategies. The comparison with AdaBoost and SVM shows the benefits of exploiting contextual information in discriminative approaches while the comparison with a binary CRF justifies the use of a multi-class framework.

A novel method for automatic lumen detection based on multi-class classification on IVUS images has been presented integrating in the same framework CRF, ECOCs and deformable models. The contextual information on longitudinal cut images has been used, together with a long term regularization, in order to solve problems due to the corruption in the vessel appearance. The comparison with other state-of-the-art methods clearly shows the superiority of a discriminant multi-class contextual model, resulting in the most accurate segmentation.

Radial artery volume computation can be easily performed by means of the proposed method, allowing to study the effect of different drugs administration. The promising results on the contextual-based classification of arterial vessel suggest to deeply investigate its applicability to IVUS image analysis. The proposed methodology could also be applied to coronary artery lumen segmentation and can represent the basis for accurate assessment of vessel border properties. 


\section{References}

1. Cardinal, M.H.R., Meunier, J., Soulez, G., Maurice, R.L., Therasse, E., Cloutier, G.: Intravascular ultrasound image segmentation: a three-dimensional fastmarching method based on gray level distributions. TMI 25(5), 590-601 (2006)

2. Brusseau, E., de Korte, C.L., Mastik, F., Schaar, J., van der Steen, A.F.W.: Fully automatic luminal contour segmentation in intracoronary ultrasound imaging-a statistical approach. TMI 23(5), 554-566 (2004)

3. Sonka, M., Zhang, X., Siebes, M., Bissing, M.S., Dejong, S.C., Collins, S.M., McKay, C.R.: Segmentation of intravascular ultrasound images: a knowledge-based approach. TMI 14(4), 719-732 (1995)

4. Unal, G., Bucher, S., Carlier, S., Slabaugh, G., Fang, T., Tanaka, K.: Shape-driven segmentation of the arterial wall in intravascular ultrasound images. TITB 12(3), 335-347 (2008)

5. Rotger, D., Radeva, P., Fernández-Nofrerías, E., Mauri, J.: Blood detection in ivus images for $3 \mathrm{~d}$ volume of lumen changes measurement due to different drugs administration. In: CAIP, pp. 285-292 (2007)

6. Lafferty, J., Mccallum, A., Pereira, F.: Conditional random fields: Probabilistic models for segmenting and labeling sequence data. In: Proc. 18th ICML, pp. 282289. Morgan Kaufmann, San Francisco (2001)

7. Dietterich, T.G., Bakiri, G.: Solving multiclass learning problems via errorcorrecting output codes. JAIR 2, 263-286 (1995)

8. Macqueen, J.B.: Some methods of classification and analysis of multivariate observations. In: Proceedings of the Fifth Berkeley Symposium on Mathematical Statistics and Probability, pp. 281-297 (1967)

9. Bovik, A.C., Clark, M., Geisler, W.S.: Multichannel texture analysis using localized spatial filters. TPAMI 12(1), 55-73 (1990)

10. Ojala, T., Pietikäien, M., Mäenpää, T.: Multiresolution gray-scale and rotation invariant texture classification with local binary patterns. TPAMI 24(7), 971-987 (2002)

11. Demi, M., Bianchini, E., Faita, F., Gemignani, V.: Contour tracking on ultrasound sequences of vascular images. PRIA 18(4), 606-612 (2008)

12. Vishwanathan, S.V.N., Schraudolph, N.N., Schmidt, M.W., Murphy, K.P.: Accelerated training of conditional random fields with stochastic gradient methods. In: ICML 2006, pp. 969-976. ACM, New York (2006)

13. Schapire, R.E.: The boosting approach to machine learning: An overview (2002)

14. Kumar, S., Hebert, M.: Discriminative fields for modeling spatial dependencies in natural images. In: Advances in Neural Information Processing Systems (2003)

15. Yedidia, J.S., Freeman, W.T., Weiss, Y.: Understanding belief propagation and its generalizations, 239-269 (January 2002)

16. Escalera, S., Pujol, O., Radeva, P.: On the decoding process in ternary errorcorrecting output codes. TPAMI 99(1) (2009)

17. Pujol, O.: A semi-Supervised Statistical Framework and Generative Snakes for IVUS Analysis. PhD thesis, Autonomous University of Barcelona (2004)

18. Boser, B.E., Guyon, I.M., Vapnik, V.N.: A training algorithm for optimal margin classifiers. In: COLT, pp. 144-152 (1992)

19. Rifkin, R., Klautau, A.: In defense of one-vs-all classification. JMLR 5, 101-141 (2004) 